\title{
Analysis on Essential medicines' use and its influencing factors in Beijing's Community Health Institutions
}

\author{
Yu Yang ${ }^{1}$ \\ ${ }^{1}$ Department of pharmacy, Beijing Union University Hospital, Beijing, China
}

Keywords: Essential Medicine; Community Health Institutions; Application Status; Analysis.

\begin{abstract}
To evaluate the use of essential medicines and its influencing factors in community health institutions and analyze problems in the stock and use of essential medicines so that we can provide references and suggestions for the Essential Medicine System. Methods The stock status of essential medicines and relevant problems in community health service institutions were investigated. Results and Conclusion Both the list and supply of essential medicines could not meet the clinical need of patients at community health centers. First-aid and low-priced essential medicines were out of stock. The low-priced essential medicines trigger both the patients and medical staff's concerns on drug quality. The methods of evidence-based practice should be applied in each field that implement the essential medicines system. With the help of good policy planning, we can solve the problems in essential medicines and provide better service for the public.
\end{abstract}

\section{Introduction}

Beijing's health resources are in the leading position in China, but the use of medical resources at all levels of hospitals is uneven and it is difficult to be improved. The core problems are the lack of hierarchical diagnosis system and unbooked hospital visits[1]. The establishment and implementing of hierarchical diagnosis system could alleviate the contradiction between supply and demand in health service effectively[2].

Therefore, in recent years, the government takes the development of community health service as a breakthrough for the reform of urban health system. Together with the launch of the new national list of essential medicines [3] as an important key and implementing drug sales "zero-slip" policy[4], we can solve the problem of "difficulty and high costs of getting medical service".

In March 2010, 324 community health service centers in Beijing began to implement the national essential medicine system. Essential medicines are on sale in all these centers which must be the first choice and reach a certain proportion of use.

This study aimed to explore the influencing factors that affect the use of essential medicines in community health institutions. The results of this study would make essential medicine list meet the clinical need better, such as cutting drug costs and improving the quality of health care, increasing patient's consultation rate so that community health resources could be utilized.

\section{Study design}

Materials. In this study, 2 community health service centers with relatively more outpatient visits from each of Beijing's six urban districts and Huairou district were selected and 6 community health service stations from central urban areas were chosen as well. The condition of supply was studied in these health centers. Statistics from Beijing Municipal Health Bureau were collected before and after the implementation of the essential medicine system (2009- 2015) and they were summarized.

Qualitative analysis was conducted through semi-structured interviews. Three directors of community health service centers, seven directors of pharmacies and 10 pharmacists who knew the circulation of drugs well were interviewed to discover the problems relating to essential medicines.

Data analysis methods. Excel 2013 was used for quantitative data statistics and analysis, and qualitative data were recorded into documents on the spot and category analysis was applied. 


\section{Result and analysis}

Status of species of the essential medicines list. The 2012 edition of National Essential Medicines List (primary health institutions section) contained 520 species of essential medicines[5]. In addition, another 179 species that were not in the National Essential Medicines List originally were added to the list by Beijing Municipal Bureau of Health (for community health institutions' use) and they were put into Beijing community drug procurement catalog. Therefore, at present Beijing essential medicines directory contains 699 species, including 379 western drugs and 320 Chinese medicines.

Table 1 The numbers of essential medicines in stock in community health institutions in September

\begin{tabular}{llllll}
\multicolumn{2}{c}{$2014(\bar{X})$} \\
$\begin{array}{l}\text { Species on the } \\
\text { essential } \\
\text { medicine list }\end{array}$ & $\begin{array}{l}\text { Number of } \\
\text { community } \\
\text { center }\end{array}$ & $\begin{array}{l}\text { Species of } \\
\text { essential } \\
\text { medicines in } \\
\text { stock in } \\
\text { September }(\bar{X})\end{array}$ & $\begin{array}{l}\text { Percentage of } \\
\text { the essential } \\
\text { medicine list } \\
/ \%\end{array}$ & $\begin{array}{l}\text { Species of } \\
\text { western } \\
\text { medicine }\end{array}$ & $\begin{array}{l}\text { Species of } \\
\text { Chinese } \\
\text { medicine }\end{array}$ \\
\hline 699 & 14 & 377 & $53.9 \%$ & 186 & 191
\end{tabular}

The field survey in September 2014 showed the stock of essential medicines accounted for 61.6\% of the total number of drugs in the institutions. This demonstrated that community health institutions purchased non-essential medicines for outpatient service to meet the needs of patients. Essential western medicines in stock in the institutions accounted for $49.1 \%$ and $59.7 \%$ of traditional Chinese medicines on the list. These low percentages, especially the western medicine (less than 50\%), showed that some drug types in the essential medicines directory were rarely used by community health institutions.

Analysis of essential medicines in short supply. The out-of-stock essential medicines in 14 community health centers and six stations were studied and analyzed. The data showed there were 111 types of essential medicines with at least 157 drugs that were in short supply, including 27 types of emergency medicines. Among the out-of-stock medicines, 51 types of western medicines and 22 types of Chinese medicines were in the low-priced medicine directory released in 2014 by the National Development and Reform Commission. Together, these accounted for $65.8 \%$ of total medicines. Among these low-priced medicines, 17 species were emergency medicines. It illustrated that the low price of essential medicines caused the inadequate supply of essential medicines. [6]

Problems in the use of essential medicines. Interviewing the directors and other personnel at the centers we got to know that some well-known pharmaceutical enterprises failed in essential medicine bidding in 2013, and that was why some community health institutions could not use the domestically produced drugs with better efficacy as well as some imported brands. The available alternatives were essentially less well-known domestic brands with lower price. Patients were reluctant to use those medicines.

Another concern from patients was the quality of drugs with a low bid price. For example, captopril produced by a certain manufacturer recently withdrew from the market due to its quality. Patients also found some nifedipine and nitroglycerin unacceptable because of their therapeutic effects.

The low-priced essential medicines also trigger medical staff's concerns on drug quality, particularly for injections. Medical staffs are highly worried about their professional duties and potential liability for occurrence of adverse events caused by sub-standard injection of drug. Most community health service stations have no facilities for handling emergency. At the same time, the increasing number of doctor-patient disputes made physicians reluctantly use infusion products, and they often advised patients to be transferred to a hospital for better treatment. This deviated from the original intention of essential medicines system. 


\section{Discussions}

Because of the inconsistency between essential medicines directory and drugs prescribed in community medication in the use of essential medicines in the community medical institutions, shortage in the supply of essential medicines, and lack of trust in brand recognition and quality of essential medicines and other aspects, both the catalogue and the supply of essential medicine can not meet the needs of patients, and the functions of providing medical security and reducing the drug expense have not all play out. These problems hindered the essential medicines system from implementing smoothly and, weakened its role in improving health care quality as well as increasing drug expenses[7]. Some essential medicine policies in practice have restricted the service capacity of community health institutions[8], and they also affected the function of community medical institutions[9].

The main reasons behind the problems lie in:

First, the applicability and pertinence of the Essential Medicine Directory is limited, and the directory updates not timely.

Community medical institutions mainly treat common diseases and frequently occurring diseases. Hence drugs for treating less frequently occurring disease or severe disease in the Essential Medicine List are rarely used in community medical institutions.

Part of medicines in the directory does not conform to the actual demand of city residents: drugs for community use are not synchronized with those in Class-2 or Class- 3 hospitals. Thus the referral patients can not achieve follow-up drug therapy in the community; besides, some drugs which have more adverse reactions or are rarely used now fail to update.

Community medical institutions have to stock some non-essential medicines which are commonly used by patients to increase outpatient visits .

Essential Medicine Directory serves for all the patients, while community health services mainly for local residents with the elderly, chronic patients, women, children and the disabled. These two target groups are not completely coincident.

Both the inadequacy and rare use of certain medicines on the Essential Medicines List would have negative effect on the service quality of community health service institutions[10]. A study made by $\mathrm{Li}$ and Wang on patients' satisfaction with the use of essential medicines also showed that most patients wanted to increase essential medicine species when they answered the questionnaire[11].

Therefore, we should take full account of the classification and the incidence rate of the disease, the characteristics of the patient group and so on. And the Essential Medicine List should be updated in time with medicines having better curative effect and less adverse reactions.

Second, the forward-looking design of the system is insufficient and it ignored the manufacturer's profit game behavior.

The short supply of essential medicines was caused by the current centralized drug bidding procurement. The procurement in Beijing brought down the purchase price by about 26\%[12]. Low-priced medicines reduced the burden of consumers for a short time. But each time after the bidding, a number of commonly-used drugs with low prices would disappear from clinical use. Profits are the lifeline of enterprises [13], and therefore many enterprises that won the bid are reluctant to produce. What is more, some high-quality enterprises even withdrew from bidding which had an impact on the supply and the quality of essential medicines. And this in turn affected the normal operations of community medical institutions.

The price of essential drugs should be released properly as a lever to adjust the market. Moreover, effective measures are insufficient to ensure the production and supply of less dosage but clinical necessary drugs. For emergency medicine, the government lack subsidies, tax breaks and other policies.

Third, The List pays more attention on the basic health care while neglect higher levels of demands by patients. 
The reason why some essential medicines lacked brand recognition was that, in China, lots of patients evaluated the brand of medicines according to the prices. Most Chinese consumers think that the higher the price, the better the efficacy. Hence, patients often demand more expensive drugs in community health service institutions[14]. This poor perception among consumers was supported by the study made by Chen Juan et al [15]. The survey of residents' attitudes towards community health service in Beijing showed that patients were not satisfied with essential medicines.

Patients and medical personnel were also concerned about the quality of essential medicines. Theoretically, essential medicines have been used in clinical application for many years and they were safe and effective. But their safety was merely based on the awareness of adverse reactions and their production process. Since quality assurance system for essential medicines has not been established yet, safety related to the quality of medicines could not be guaranteed[16]. This safety concerns was further exacerbated by the fact that during the process of evaluation, price was often the decisive factor to get the bid[17], other important factors, such as the size and past reputation of the bidding enterprise, quality of the products were ignored. This emphasis on "low price" could lead to the low quality and efficacy.

The biding system of essential medicines determines bid by generic names, hence it emphasizes on functions of cheap prices and basic medical security. However, many patients pay more attention on drugs of recognized brand or good efficacy and high safety. Hence the bidding behavior hasn't focus on medical consumption psychology sufficiently. Instead of relying on the price factor, essential medicine centralized purchasing should be more quality-oriented[18]. The functions, characteristics, market, policy and other information of essential medicines required should be fully understood by the bidding system practitioners. Through these efforts, the brand recognition and quality trust on essential medicines by patients would be improved to enable the community drug directory to be more attractive.

\section{Recommendations}

In view of above, the methods of evidence-based practice should be applied in each field that implement the essential medicines system such as employing the best current research evidences, focusing on the clinical experience and evaluation, and giving consideration to both values and desires of patients about essential medicines to promote their utilization.

Therefore, this article puts forward following proposals:

Update drugs in the directory rarely used by community, and supplement those commonly used by patients into the directory to improve its applicability.

For the essential medicines of small dosage, low price and clinical necessity which are easily in shortage, government intervention should be applied to strengthen their commitments to social responsibility, and to subsidize and award the enterprises who complete the production tasks.

The design of centralized bidding system of drugs need to be more flexible such as establishing prior channels for manufacturers of high quality or brand drugs with historical precipitation to encourage these enterprises' bidding in the centralized procurement.

Establish drug feedback and scoring information system in community health institutions, thus patients can make a comprehensive evaluation on indexes such as medicine's quality, efficacy, convenience, price, and so on. By reference of these scores, the essential medicine update and elimination mechanism could be established.

With better policy planning and coordinated efforts from all parties, the essential medicine system will improve health care quality and serve the public better in the near future.

\section{Acknowledgement}

This work was supported by New Start Program of Beijing Union University under Grant number Zk10201611. 


\section{References}

[1] Wang Hufeng, Wang Hongyun, Thought on issues related to the building of grading diagnosis and treatment system[J]. China Medical Administration Sciences. 2014,4 (1): 28-30

[2] Fu Qiang, Strategic Choice for Promoting Hierarchical Treatment Model[J]. Chinese Health Economics, 2015,34(2):28-31

[3] National Health and Family Planning Commission. The National Essential Medicine List (2012 edition) is released [EB/OL]. (2013-03-15)[2015-06-09]. http:/www.nhfpc.gov.cn/yaozs/s3578/ 201406/07a8e8930f4e4c3d8e4e03412fe6b3a8.shtml.

[4] National Health and Family Planning Commission. Suggestions for the implementation of establishing the National Essential Medicine System [EB/OL]. (2009-08-18)[2015-06-05]. http://www.nhfpc.gov.cn/tigs/s9660/200908/98b25d019fdb4700b3409daf43f8bd81.shtml.

[5] National Health and Family Planning Commission. The National Essential Medicine List (2012 edition) (Order No. 93 of the Ministry of Health) [EB/OL]. (2013-03-15)[2015-05-15]. http://www.moh.gov.cn/mohywzc/.

[6] Fang Zhang, , Yu Yang, Shu Chuen Li. Analysis of the Utilization Status of Essential Medicines in Beijing's Community Health Institutions $t[\mathrm{~J}]$. Therapeutic Innovation \& Regulatory Science, 2016,(8):1-6

[7] Ju Ting, Chen Yongfa. The function of the Essential Medicine System to the Issue of High Drug Cost[J]. Chinese licensed pharmacists, 2011,8(4):45-48

[8] Beijing Health and Family Planning Commission. The reform of developing community health service steadily is deepened continuously[EB/OL].(2014-01-15)[2015-02-09]. http://www.bjhb.gov.cn/wsxw/201401/t20140115_70379.html

[9] Li Dongfeng, Wang Yirui, Wang Jianhua, et al. Analysis of current administration of essential drugs and study on corresponding coping measures for medical institutions in Xinjiang Area[J]. Chinese Journal of Hospital Pharmacy, 2015,35(7):647-648

[10] Tang Shengchun, Chang Xing, Liu Chunsheng, et al. Research on the influence and countermeasures of the implementation of national essential medicine system in terms of supply and demand on community health services[J]. Chinese Journal of Health Policy, 2010,3(12):8-13

[11] Li Ying, Wang Hufeng. Research on Implementation of National Essential Medicine System in Beijing[J]. Chinese Hospital Management, 2014;34(4):49-51

[12] Beijing Health and Family Planning Commission. Beijing essential medicine centralized purchasing prices fell by 26\%, could reduce expenses at 973 million Yuan a year [EB/OL]. (2013-04-09) [2015-02-20]. http://www.bjhb.gov.cn/wsxw/201304/t20130409_59181.html

[13] Li Jun, Xi Xiaoyu, Chu Shuzhen. Influence of Medicine Interest Group on the Essential Drug Accessibility[J]. Chinese Journal of Pharmaceuticals, 2011,42(12): 107-110

[14] Guo Gong, Sun Zhenqiu, He Qiong, et al. Problems and Suggestions for the Essential Medicine System in China[J]. Journal of Central South University (social science), 2012, 18(3): 31-36

[15] Chen Juan, Ni Jing, Fang Kai, et al. Survey on satisfaction of community health service among residents in a district of Beijing[J]. Chinese Journal of Health Policy, 2012,5(1):28-31

[16] Peng Lu, Guo Jing, Chen Dandi. Effects of Essential Drug System on Primary Medical Institutions[J]. China Pharmacy, 2010,21(32):2996-2998

[17] Li Jun, Qiu Feng. Exploration of Balance between Pharmaceutical Quality and Price during

Drug Bidding[J]. China Pharmacy, 2013,24 (4):319-321 
[18] Yu Xiaoyan, Tang Shaolian. Study on correlation between quality and price in bidding procurement for essential drugs[J]. China Medical Herald,2015,12(8):105-108 